\title{
Optic nerve head segmentation using fundus images and optical coherence tomography images for glaucoma detection
}

\author{
T. R. Ganesh Babu ${ }^{\text {a }}$ S. Shenbaga Devi ${ }^{\mathrm{b}}$, R. Venkatesh ${ }^{c}$
}

Background. Glaucoma is a common causes of blindness. The associated elevation in intra ocular pressure leads to progressive degeneration of the optic nerve and resultant structural changes with functional failure of the visual field. Since, glaucoma is asymptomatic in the early stages and the associated vision loss is irreparable, its early detection and timely medical treatment is essential to prevent further visual damage.

Objective. This paper presents a novel method for glaucoma detection using digital fundus image and optical coherence tomography (OCT) image.

Method. The first section focuses on the features such as cup to disc ratio (CDR) and the inferior superior nasal temporal (ISNT) ratio which were obtained from fundus images. The above features were used for classifying the normal and glaucoma condition using back propagation neural network (BPN) and Support Vector Machine (SVM) classifiers. In the second part of the article, features such as CDR and two novel features, cup depth and retinal thickness were obtained from the OCT image. These features were evaluated by the BPN and SVM classifier.

Results and Conclusion. The combined features from fundus and OCT images were analyzed. The system proposed here is able to classify glaucoma automatically. The accuracy of BPN and SVM Classifiers was $90.76 \%$ and $96.92 \%$ respectively.

Key words: glaucoma, optic disc, optic cup, blood vessels, neuro retinal rim, cup depth, retinal thickness

Received: June 9, 2015; Accepted: September 30, 2015; Available online: October, 23, 2015

http://dx.doi.org/10.5507/bp.2015.053

${ }^{a}$ Department of Electronics and Communication Engineering, Shri Andal Alagar College of Engineering, Chennai, Tamilnadu, India ${ }^{b}$ Department of Electronics and Communication, Anna University, Chennai, India

'Aravind Eye Hospital, Tamilnadu, India

Corresponding author:T.R.Ganesh Babu, e-mail:ganeshbabutr@gmail.com

\section{INTRODUCTION}

Glaucoma is one of the common causes of blindness. It causes progressive degeneration of optic nerve fibers and leads to structural changes of the optic nerve and a simultaneous functional failure of the visual field. Since, glaucoma is asymptomatic in the early stages and the associated vision loss cannot be restored, its early detection and subsequent medical treatment is essential to prevent further visual damage ${ }^{1}$.

A significant feature of glaucoma is the absence of warning symptoms. Glaucoma diagnosis is mainly focused on detecting changes in the visual functioning of the eye at early stages of the disease and vision can be protected through medical treatment. $98 \%$ of visual loss can be prevented by timely diagnosis. The shortage of trained personnel,however leads to the need for an automatic retinal image analysis system. Our research was aimed at detecting glaucoma using digital images to assist the ophthalmologist and save time in screening and,diagnosing glaucoma. Fundus and OCT images are types of images used by ophthalmologists to detect glaucoma. In the fundus image, there is a small depression seen at the front of the optic nerve head. The depression is known as the optic cup and its diameter is smaller than the diameter of the optic disc. As glaucoma progresses, neural tissues die, the nerve fiber layer thins, and the Cup to Disk Ratio (CDR) increases. Hence, the CDR is one of the features used to detect glaucoma. In addition to CDR, a parameter called the ISNT ratio which is the ratio of the sum of the blood vessels in the Inferior and the Superior regions to the sum of the blood vessels in the Nasal and the Temporal region is used $\mathrm{d}^{2}$.The same parameter is also used based on the neuron retinal rim area. In OCT, the cup region will show features related to glaucoma. Hence, these features are also used for the detection of glaucoma.

The aims of research were to:

1. determine the CDR and ISNT ratio from retinal fundus images

2. determine the features namely CDR, cup depth and retinal thickness in Optical Coherence Tomography (OCT) images.

3. classify the glaucoma automatically using the Back Propagation Neural Network (BPNN) and the Support Vector Machine (SVM).

4. determine the efficiency of detection, the methods used had to be compared on the basis of classification accuracy as apercentage.

Features such as the CDR and ISNT ratio obtained from fundus images are used to detect glaucoma. In this section different techniques for acquiring the above features that are available in the literature are discussed.

The features such as CDR, cup depth and retinal thickness are used to detect the glaucoma using the OCT im- 
age. In this section, different techniques which are used for segmentation of optic nerve head in OCT image that are available in the literature are discussed.

Artemas Herzog et al. ${ }^{3}$ provided a hybrid edge-supported boundary detector that finds the contour separating the retina and optic nerve head (on one side) from the vitreous humor on the other. They then partitioned this boundary using a model-based technique to identify the optic cup. The lower retinal-choroid boundaries are extracted using a Markov model. Boyer et al. ${ }^{4}$ presented their results on optic nerve head segmentation and geometric characterization through OCT data. They developed a complete, autonomous algorithm based on a parabolic model of cup geometry and an extension of the Markov model introduced to segment the retinal-nerve head surface to identify the choroid-nerve head boundary.

An overview of the detection of glaucoma using fundus and OCT images carried out by previous researchers has been presented. A literature review allows the following inferences:

1. The level set based algorithm generally needs human interaction. The detection of incorrect boundaries by the conventional level set method is avoided by continuously reinitializing the level set function throughout the evaluation process.

2. Variation level set method is introduced to eliminate the re-initialization procedure.

3. The energy minimization based variation level set method does not consider the edge and region based information. Hence the segmentation of optic cup is very difficult.

4. Due to large intensity variations in the cup region, thresholding techniques are not adequate.

5. Segmentation of OD and OC using mathematical morphological techniques greatly depend on the shape and size of the selected structuring elements.

6. Active contour modelling takes more convergence time and snakes capture limited range of initialization around the region of interest. The active contour model takes more convergence time and snakes capture limited range of initialization around the region of interest Ganeshbabu et al. ${ }^{5}$.

7. CDR determination is difficult for diabetic patients with glaucoma because the cup and disc boundaries are merged.Siddalingaswamy et al. ${ }^{6}$.

8. Exudates are formed in diabetic patients. Since the pixel intensity of the exudates and optic disc are similar, detection of the optic disc is difficult.

In order to strengthen the glaucoma examination for mass screening, the fundus image features such as CDR, ISNT ratio for both neuro retinal rim and blood vessels and the OCT image features such as CDR, cup depth, retinal thickness are combined in the proposed method.

Liu et al. ${ }^{7}$ presented a variation level set method to automatically extract the optic disc. For the optic cup, two methods making use of color intensity and threshold level set are evaluated. Zhang et $a .^{8}$ presented segmentation and boundary detection. A fused approach is proposed based on multimodalities including level set segmenta- tion, convex hull and ellipse fitting boundary smoothing. Wong et al. ${ }^{9}$ discuss ARGALI, an automated system for glaucoma detection which, employs level set methods for segmenting the optic cup and disc from retinal images, combined using a fusion network, to determine the cup to disc ratio (CDR). The system uses SVM as an alternative fusion strategy in ARGALI, and evaluates its performance against the component methods and neural network (NN) fusion in the CDR calculation. Nayak et al. ${ }^{10}$ describe a novel method for glaucoma detection using digital fundus images. Digital image processing techniques, such as preprocessing, morphological operations and thresholding, are widely used for the automatic detection of optic disc, blood vessels and computation of features. Features such as CDR, shift of the optic nerve head and ISNT are extracted. These features are validated by classifying the normal and glaucoma images using neural network classifier. Joshi et al. ${ }^{11}$ used a method for optic disc and cup segmentation from monocular color retinal images for glaucoma assessment. Here Active contour model was used for robust OD segmentation. Cup segmentation is done using r-bends information. Finally, the cup boundary is obtained using local spline interpolation on the detected r-bends. Mishra et al. ${ }^{12}$ explain the active contour method to be used to determine the CDR from the color fundus images to investigate thepathological process of glaucoma. Ho et al. ${ }^{13}$ developed an automatic detection system which hastwo major phases: the first phase performs series modules of digital fundus retinal image analysis including vessel detection, cup to disc ratio calculation, and neuro-retinal rim for ISNT rule. The second phase determines the abnormal status of retinal blood vessels from a different aspect.

\section{METHODS AND MATERIALS}

\section{Detection of glaucoma using retinal fundus images}

The cup to disc (CDR) ratio is an important indicator of the risk of the presence of glaucoma in an individual. In this study, two methods to calculate the CDR from fundus images are presented.

\section{Segmentation of optic disc and optic cup}

In order to compute CDR, the diameter of optic cup and optic disc should be calculated. In this method, $\mathrm{K}$-means clustering technique is used to extract the optic disc in an automatic manner ${ }^{14}$ the $\mathrm{k}$ value is automatically selected by Hill climbing algorithm. For the cup, a method by making use of Fuzzy C-Mean (FCM) clustering is evaluated. The method is as follows:

The Green plane $(G)$ from the RGB fundus image is separated and it is considered for the analysis because $G$ plane provides better contrast than the other two planes ${ }^{15}$. Then the maximum brightest point within the optic disc in the $\mathrm{G}$ plane is determined. An approximate region of a size of 360x360 pixels is selected around this identified brightest point. This region is segmented using K-mean clustering algorithm initially. The segmentation accuracy of optic disc mainly depends on the number of clusters 
( $\mathrm{K}$ value) used in the $\mathrm{K}$ means clustering algorithm. Due to intensity variations in the optic cup, optic disc, blood vessels and background, the number of clusters cannot be predicted. Hence, Hill-climbing algorithm ${ }^{16}$ is used to determine the optimal number of clusters by identifying peaks in the 3D colour histogram of Lab colour channel. After obtaining the contours, an elliptical fitting ${ }^{17}$. Table 1 step is introduced to smooth the obtained optic disc boundary.

Compared to the extraction of the optic disc, optic cup segmentation provides an even greater challenge. The optic cup region is masked in many places by the blood vessels emerging through the optic disc. Hence to correctly identify the region of optic cup, the blood vessels have to be removed from this region. To blur the blood vessels, wavelet transform and morphological closing operation are used and K-means clustering is performed by assigning $\mathrm{k}$ equal to 3 to detect optic cup. This result includes not only optic cup but also some small extra regions and this affects the CDR value. Therefore the FCM clustering is applied to the image to overcome the above drawback ${ }^{18}$. The optic cup boundary is smoothed by elliptical fitting and also by the morphological technique. The CDR ranges and mean error of the proposed method are shown. In the proposed system, 50 normal and 50 glaucoma images are used with specified CDR values. For these images, the gold standard values of CDR specified by the ophthalmologist are available. This CDR range for a normal image lies between 0.15 0.35 and for glaucoma images, it lies between $0.4 \sim 0.9$. The mean error is calculated by comparing CDR values obtained by the proposed system with gold standard values.

The optic cup region obtained from FCM does not show the complete contour ${ }^{19}$ and hence error is seen in the cup-to-disc ratio. To increase the accuracy, another algorithm for segmentation of optic disc and optic cup in fundus image is developed.

\section{Glaucoma detection using Spatially Weighted Fuzzy C Mean Clustering}

In this method, the optic disc and optic cup are segmented using Spatially Weighted Fuzzy C Mean
Clustering Algorithm (SWFCM). The standard FCM does not consider the spatial information of pixels and in turn, the segmentation result is affected. One important characteristic of an image is that neighboring pixels are highly correlated which is considered in the SWFCM method. Hence for cup and disc segmentation, SWFCM is applied ${ }^{20}$, Anapproximate region of interest of size $360 \times 360$ pixels is selected around the brightest point in the green $(\mathrm{G})$ plane of the fundus image. The ROI image contains not only the optic disc and cup region but also the blood vessels. Hence the blood vessels must be removed in order to obtain better segmentation of optic disc and cup region. To blur the blood vessels, wavelet transform and morphological closing operation are used.

SWFCM is applied to the images in which blood vessels are already blurred. The result of SWFCM will have clusters corresponding to optic cup, disc and back ground. After extracting the optic disc and cup from the clusters, elliptical fitting is applied for disc region as the shape of the disc is round or vertically slightly oval disc. For optic cup, elliptical and morphological techniques are applied independently to smooth the boundary. Table 2 shows the CDR ranges and mean error of the proposed method using fundus images. The algorithm is applied to the same image set used for the FCM and K means methods.

Fig. 1 (a) and 1 (b) show the segmented and elliptically fitted optic cup and disc for normal and glaucoma conditions respectively. Fig. 2 (a) and 2 (b) show the segmented and elliptically fitted optic disc and actual contour of optic cup for the same conditions.

From Tables 1 and 2, it is observed that the SWFCM mean error is smaller than K-Mean \& FCM clusteringoutput.The other main advantage of the SWFCM method is that both optic disc and optic cup are segmented in one stage from the results of SWFCM (ref. $\left.{ }^{21}\right)$. Hence, for further analysis with CDR, both optic cup and disc are segmented applying SWFCM algorithm.

\section{ISNT Analysis}

According to the literature, the CDR range for normal images is from 0.1 to 0.3 (ref. ${ }^{22}$ ). But for the normal images processed, many images have CDR values more than

Table 1. CDR ranges and mean error of the proposed method.

\begin{tabular}{llccc}
\hline \multirow{2}{*}{$\begin{array}{l}\text { Smoothening } \\
\text { Technique }\end{array}$} & \multirow{2}{*}{$\begin{array}{l}\text { Segmentation } \\
\text { Technique }\end{array}$} & Normal & CDR Ranges & Abnormal \\
\cline { 3 - 5 } & & $0.13 \sim 0.37$ & $0.403 \sim 0.891$ & Mean error (\%) \\
\hline Elliptical method & K-means & $0.13 \sim 0.359$ & $0.402 \sim 0.881$ & 4.5 \\
& FCM & $0.12 \sim 0.329$ & $0.401 \sim 0.886$ & 3.83 \\
Morphological Method & K-means & $0.122 \sim 0.361$ & $0.403 \sim 0.866$ & 3.1 \\
& FCM & & & 3.52 \\
\hline
\end{tabular}

Table 2. CDR ranges and mean error of the proposed SWFCM method.

\begin{tabular}{|c|c|c|c|c|}
\hline \multirow{2}{*}{$\begin{array}{l}\text { Smoothening } \\
\text { Technique }\end{array}$} & \multirow{2}{*}{$\begin{array}{l}\text { Segmentation } \\
\text { Technique }\end{array}$} & \multicolumn{3}{|c|}{ CDR Ranges } \\
\hline & & Normal & Abnormal & Mean error (\%) \\
\hline Elliptical method & SWFCM & $0.291 \sim 0.348$ & $0.587 \sim 0.541$ & 3.06 \\
\hline Morphological Method & SWFCM & $0.274 \sim 0.328$ & $0.513 \sim 0.599$ & 1.67 \\
\hline
\end{tabular}


0.33. As the feature CDR overlaps for normal and glaucoma images, another feature ISNT is analyzed for glaucoma detection. Most blood vessels are concentrated in the superior and the inferior regions of the optic disc for normal conditions. A shift in the optic nerve head causes a slight increase in the area covered by the blood vessels in the nasal and temporal regions and decreases the area covered in superior and inferior regions. Therefore by taking the ratio of the sum of blood vessels area in the inferior and superior regions to sum of area of blood vessels in the nasal and temporal regions will show a difference between normal and glaucoma images.

From the fundus image, the blood vessels are segmented using the local entropy thresholding method. The detection of blood vessels for ISNT analysis is developed in the following steps:

1. The blood vessels are enhanced with the help of matched filter because blood vessels have low contrast. The image thus obtained is called matched filter response.

2. An entropy based thresholding scheme ${ }^{23}$ is used to differentiate the blood vessels and background in the matched filter response image. The spatial distribution of gray levels is considered because image pixel intensities are not independent of each other. It can well preserve the spatial structures in the binarizedimage.

3. There are still some misclassified pixels in the image. In order to produce a clean and complete vascular tree structure, the misclassified pixels are removed using length filtering. The length filtering tries to isolate the individual objects using the eight-connected neighborhood.

From the blood vessels detected image, a mask image is used to measure the area of the blood vessels in the ISNT quadrant. The mask is rotated by $90^{\circ}$ each time and is used to obtain the area covered by blood vessels in each quadrant and ISNT values are calculated for the same image set. Fig. 3 (a), (b), (c), and (d) show the blood vessels in each quadrant of the abnormal image after applying corresponding masks. The result shows that ISNT ratio values for the set of normal images range from 0.688 to 2.912 and for glaucoma images, it ranges from 0.4321 to 1.0621 .

The neuro retinal rim configuration becomes available for the diagnosis of glaucoma according to the ISNT rule ${ }^{9}$. The neural retinal rim is calculated by subtracting the area of the optic cup from the area of the optic disc. For a normal optic disc, the neural rim follows the property of decreasing thickness in an order of inferior, superior, nasal and temporal rims. Glaucoma frequently damages superior and inferior optic nerve fibers before temporal and nasal fibers, and it leads to a decrease in the superior and inferior rims and changes the order of ISNT relationship. Fig. 4(a), (b), (c) and (d) show the abnormal neuro retinal rim area in the four quadrants after the application of corresponding masks.

The result computed on the same set of images shows that ISNT values of neuro retinal rim for the set of normal images ranges from 1.2009 to 2.786 and for abnormal images, it ranges from 0.8868 to 1.2106 .

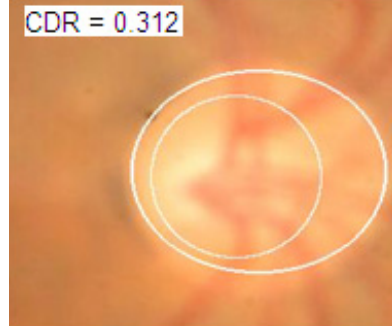

(a)Normal

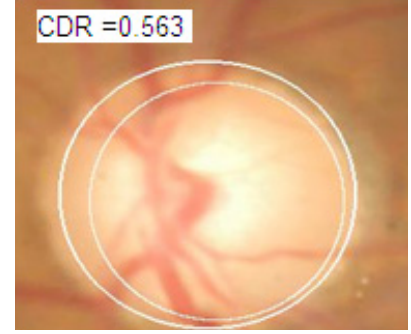

(b) Glaucoma
Fig. 1. CDR computations with elliptical fitting for optic cup and optic disc (SWFCM method).

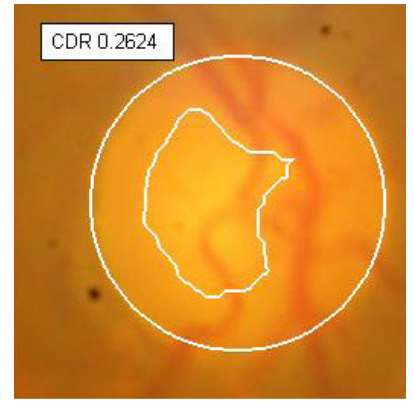

(a)Normal

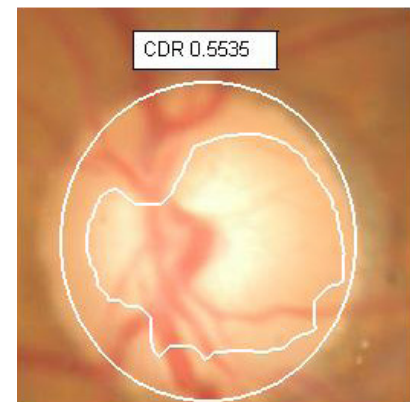

(b) Glaucoma
Fig. 2. CDR computations with elliptical optic disc and actual segmented contour for optic cup (SWFCM method).

\section{DETECTION OF GLAUCOMA BY CLASSIFIER}

Ophthalmologists are now using the CDR value from fundus image for glaucoma diagnosis. But there is an overlap of CDR values between normal and glaucoma condition, Hence to strengthen the diagnosis, in this work, two other parameters namely ISNT (rim) and ISNT ratio for blood vessels are computed by algorithms developed here. However,all these features have overlapping values between normal and glaucoma conditions. Hence it is inferred that one feature alone will not help in the diagnosis and as the next part of the work, all these features are collectively used Since normal and the specific values for glaucoma condition overlap, simple thresholding technique cannot be applied for the detection of glaucoma. Hence back propagation neural network is used for classification. In this study one hundred and fifty fundus images were used in which 50 were from normal and 100 were from glaucoma patients. Of the 50 normal images, 35 images were used for training the network and the remaining 15 were used for testing the network. In glaucoma conditions 50 images were used for testing and training the network.

\section{Back Propagation Neural Network}

The network design involves developing a three layered feed forward neural network which includes $\mathrm{N}$ neurons in the input layer where $\mathrm{N}$ is the number of inputs, $(2 \mathrm{~N}+1)$ neurons in the hidden layer ${ }^{24}$ and $\mathrm{N}_{2}$ neurons in the output layer. In this study, the number of inputs was 3 , namely 
CDR, ISNT for neuro retinal rim and ISNT for blood vessels. The number of nodes in the hidden layer was chosen as 7 and output layer had a 1 node. The proposed features CDR, ISNT ratio for neuro retinal rim area and ISNT ratio for blood vessels were calculated for all the training images and stored in an array. This array is called as the Feature base. During the training process, the difference between the output vector and the target vector is calculated and the weights are updated based on the difference value. In the proposed BPN, log sigmoid activation function is used.The log sigmoid activation function is given by Equation (1) $\left(\right.$ ref. $\left.^{25}\right)$.

$$
\log \operatorname{sig}(n)=\frac{1}{(1+\exp (-n)}
$$

Mean square error was set to 0.001 , the results of the classifierwas $89.33 \%$.

The classification accuracy is inferred to be less and improvement is needed to have better classification. For this reason, SVM which is assumed to be a better classifier has been adopted.

\section{Support Vector Machine}

The support vector classification approach is a relatively recent development in statistical pattern recognition. In this approach, optimal classification is achieved by maximizing the width of the empty area between two classes. The margin width is defined as the distance between the discrimination hyper surface in n-dimensional feature space and the closest training patterns: these are called support vectors ${ }^{26}$. The support vectors thus specify the discrimination function. Clearly, the ability to iden- tify the optimal discriminating hyper surface between two classes in a situation when many such separating hyper surfaces exists is a major strength of this approach and assist in dealing with the problem of over fitting during training. The original support vector classifier was developed for linear separation of two classes. This early limitation was later overcome by allowing non-linearly separable classes, non-separable classes, combining multiple 2-class classifiers to yield multiclass classification, and other extensions. In this study, linear kernel was used to map the training data into the kernel space. Quadratic programming was used in this study to find the separating hyper plane. The result of the classifier is $04.66 \%$. Other parameters namely Sensitivity, Specificity, Positive Predictive Value and Accuracy defining the efficiency of the classifier are also computed here.

Sensitivity refers to the percentage of abnormal fundus image classified as abnormal, Specificity refers to the percentage of normal fundus image classified as normal, Positive predictive value refers to how best it can detect the normal and accuracy refers to the ability of the classifier to classify correctly. Sensitivity, Specificity, Positive Predictive value and Accuracy are calculated by the Equations 2, 3, 4 and 5 respectively.Table 3shows the analysis of the classification results.

$$
\begin{aligned}
& \text { Sensitivity }=\left(\frac{T P}{T P+F N}\right) * 100 \% \\
& \text { Specificity }=\left(\frac{T P}{T P+F P}\right) * 100 \%
\end{aligned}
$$

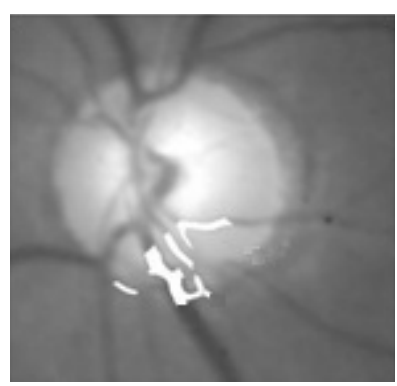

(a) Inferior side

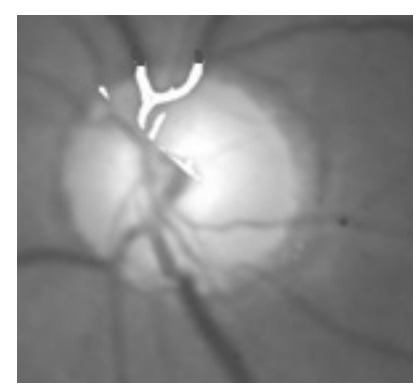

(b) Superior side

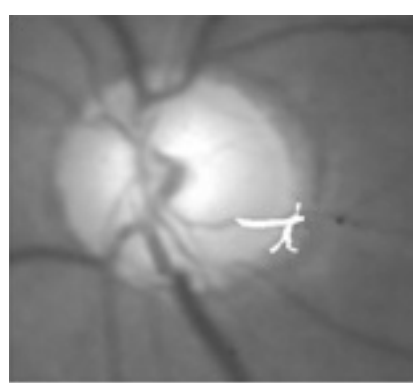

(c) Nasal side

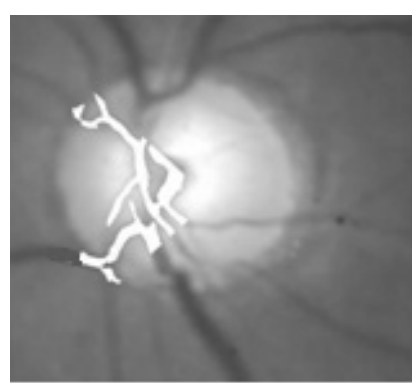

(d) Temporal side

Fig. 3. Segmented blood vessels for ab normal fundus images.

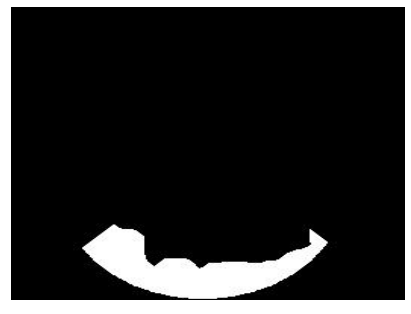

(a) Inferior side

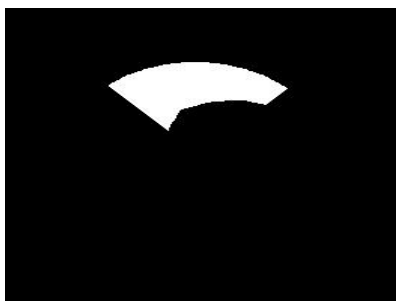

(b) Superior side

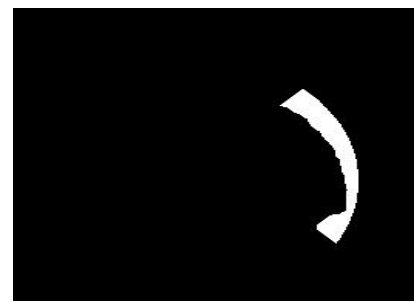

(c) Nasal side

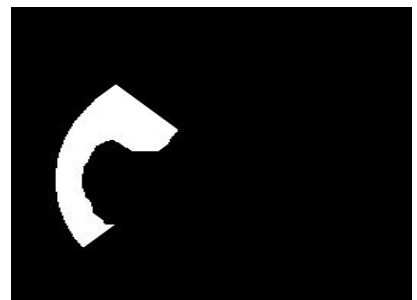

(d) Temporal side

Fig. 4. Segmented abnormal neural retinal RIM areas. 
Table 3. Analysis of classification results (Fundus image features).

\begin{tabular}{ccccccccc}
\hline Classifier & TN & TP & FP & FN & Sensitivity & Specificity & Positive Predictive value & Accuracy \\
\hline BPN & 13 & 46 & 2 & 4 & $92 \%$ & $86.66 \%$ & $95.58 \%$ & $90.7 \%$ \\
SVM & 14 & 48 & 1 & 2 & $96 \%$ & $93.33 \%$ & $97.95 \%$ & $95.38 \%$ \\
\hline
\end{tabular}

Positive Predictive Value $=\left(\frac{T P}{T P+F P}\right) * 100 \%$

Accuracy $=\left(\frac{T P+T N}{T P+T N+F P+F N}\right) * 100 \%$

TN - Normal as Normal; TP - Disease as Disease; FP - Normal as Disease; FN - Disease as Normal

From the Table 3, it is observed that SVM classifier gives better sensitivity and accuracy than BPN.

\section{AUTOMATIC DETECTION OF GLAUCOMA USING OCT IMAGES BASED ON FEATURES OF OPTIC NERVE HEAD}

\section{Determination of CDR}

In this study, two methods to calculate the CDR from OCT images are presented. In the first method, a morphological technique that extracts both the Retinal Vitreal (RV) boundary and the Retinal Choroid ( $R C$ ) boundary is investigated ${ }^{27}$. These two boundaries are used to determine the lateral extent of the optic disc and cup. The success rate of the developed algorithm was only $78 \%$ because in some images, the algorithm failed to detect the $\mathrm{RC}$ boundary. To increase the accuracy, another algorithm based on wavelet transform was developed.The retinal vitereal boundary is extracted using wavelet transform ${ }^{28}$ and region of retinal choroidboundary is extracted using multilevel thresholding ${ }^{29}$. Bezier curve fitting ${ }^{30}$ applied to smooth both RV and RC boundaries. By using RV and RC boundaries, CDR was calculated ${ }^{31}$. The OCT image of a normal subject is shown in Fig. 5 and the OCT image of glaucoma is shown in Fig. 6. The result shows that the CDR value for a set of normal images range from 0.144 to 0.308 and abnormal images range from 0.402 to 0.89 .

\section{CUP Depth Determination}

Apart from CDR, the depth of cupping also shows the condition of glaucoma. For some patients, the disc size will be smaller and hence the CDR will show a higher value which gives a false alarm. To avoid this, cup depth is also considered as another feature. By using the RV boundary, cup depth has been calculated because it increases for glaucoma condition. The OCT image of a normal subject is shown in Fig. 7. The OCT image of a subject affected by glaucoma is shown in Fig. 8. It is observed from the experimental results that cup depth in normal images lies between 740 to $1120 \mu \mathrm{m}$ and for abnormal image, it lies between 1190 to $2120 \mu \mathrm{m}$.

\section{Determination of Retinal Thickness}

Due to deeper cupping, the retinal thickness reduces in the cup region and this feature is also an indicator of

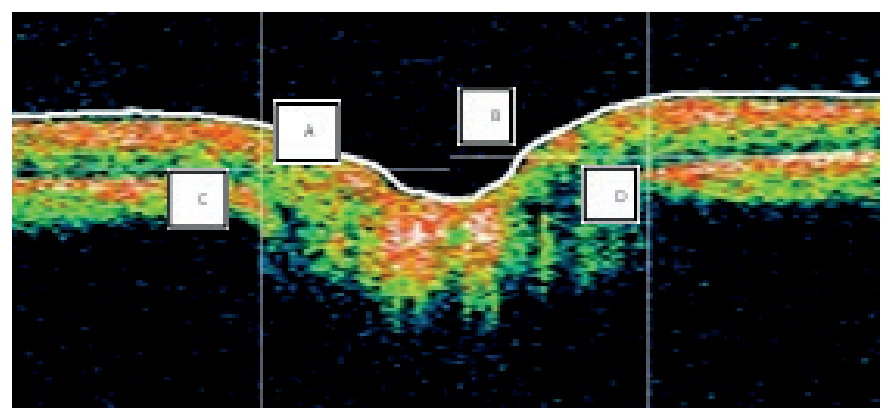

Fig. 5. OCT image of Normal subject $(C D R=0.33779)$.

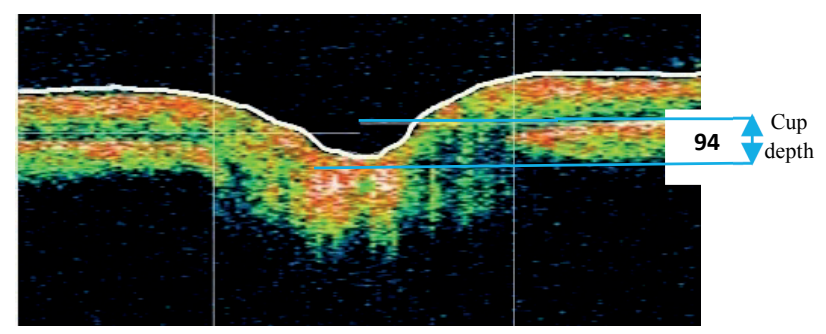

Fig. 7. OCT Normal image.

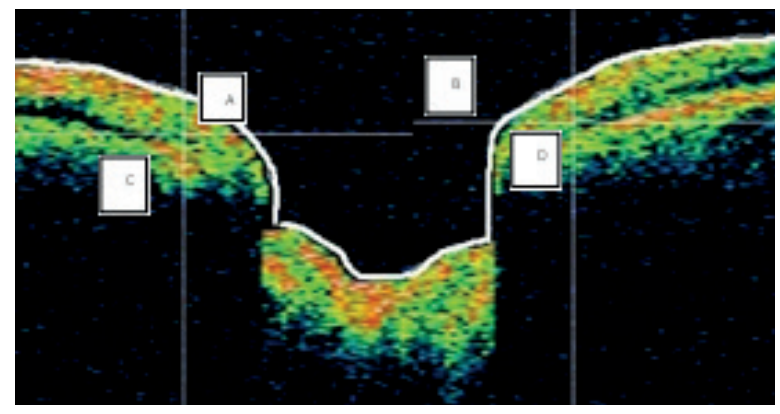

Fig. 6. OCT image of a subject affected by glaucoma (CDR $=0.76659$ ).

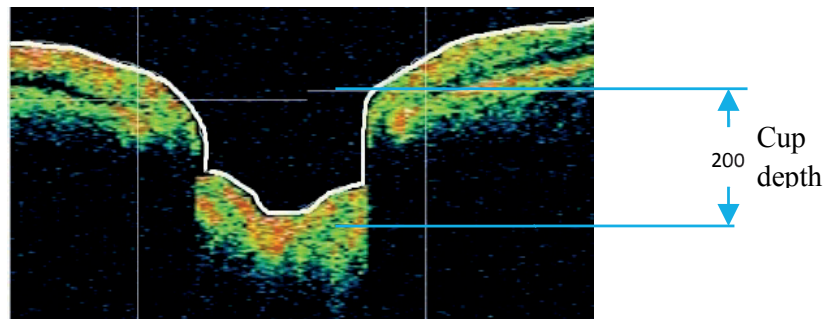

Fig. 8. OCT abnormal image. 


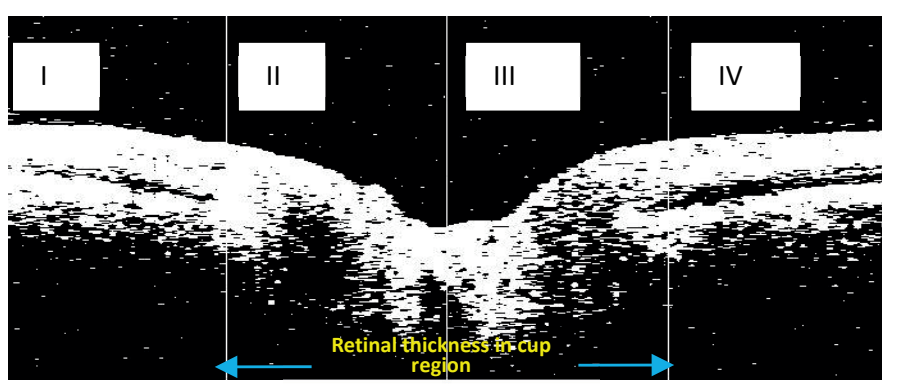

Fig. 9. Retinal area normal image.

glaucoma. Retinal thickness can be calculated from multi threshold binary image obtained from the previous phase. Fig. 9 for retinal area normal binary image and in Fig. 10 for retinal area glaucoma binary image condition. Retinal thickness is determined by calculating the number of true pixels in the second and third quadrant of the retinal area in Fig. 9 and 10. In order to determine the average retinal thickness in micron, the total number of true pixels in the regions marked as II and III multiplied with the resolution factor of the corresponding OCT camera. The resolution factor of OCT camera is $10 \mathrm{micron} /$ pixel which are used to capture the OCT images used in this work. Then the thickness measurement is given by Equation (6).

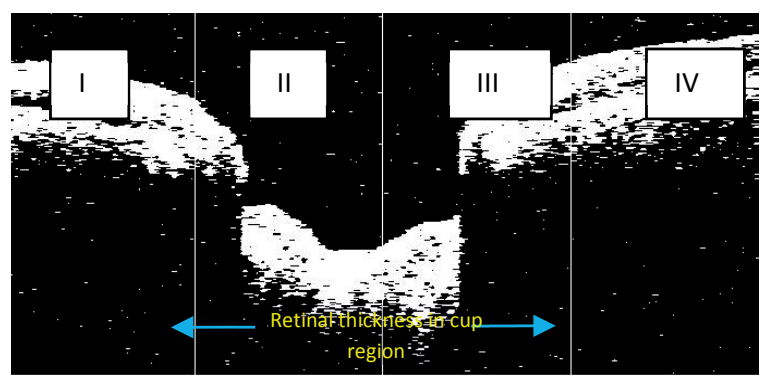

Fig. 10. Retinal area glaucoma image.

$$
\text { Re tinal Thickness in microns }=\frac{\text { resolution factor } * \text { number of pixel sin each column }}{\text { Number of column }}
$$

The result shows that retinal thickness value for the set of normal images ranges from 501.216 to $831.192 \mu \mathrm{m}$ and abnormal images ranges from 131.616 to $691.213 \mu \mathrm{m}$.

\section{Detection of Glaucoma by Classifier}

In this work one hundred and fifty OCT images are used in which 50 are from normal and 100 are from glaucoma patients. Out of the 50 normal imagesit, 35 images are used for training the network and the remaining 15 are used for testing the network. In glaucoma images 50 images are used for training and the remaining 50 images are used for testing. The parameters described in section 3.3 are used for the classifier settings for BPN and SVM classifiers. The result of the BPN Classifier is $89.33 \%$. The classifier accuracy is inferred to be less, and in order to improve it, SVM classifier is used The result of the classifier is $93.66 \%$. Other parameters such as Sensitivity, Specificity, Positive Predictive Value and Accuracy defining the efficiency of the classifier are also computed here. Table 4 shows the analysis of classification results.
From the Table 4, it is observed that SVM classifier achieves better classification accuracy than BPN.

\section{RESULTS AND DISCUSSION}

The accuracy of classification using the features of the fundus image is $95.38 \%$ and for features of the OCT image, it is $93.83 \%$. Further, details about the blood vessels are not available from OCT images while details about the retinal thickness and the level of cupping in the form of cup depth are not found in the fundus images but all these features are also important indications of glaucoma. Hence to increase the efficiency of the classification and the diagnosis of glaucoma, the features from fundus and OCT images are combined and fed into the classifiers. Glaucoma is detected by getting three features from fundus images namely CDR, ISNT for neuro retinal rim and ISNT for blood vessels. In the fundus images method, due to the merging of cup and disc region, CDR and ISNT

Table 4. Analysis of classification results (OCT image features).

\begin{tabular}{lcccccccc}
\hline Classifier & TN & TP & FP & FN & Sensitivity & Specificity & Positive Predictive value & Accuracy \\
\hline BPN & 12 & 46 & 3 & 4 & $92 \%$ & $80 \%$ & $93.87 \%$ & $89.29 \%$ \\
SVM & 14 & 47 & 1 & 3 & $94 \%$ & $93.33 \%$ & $97.91 \%$ & $93.83 \%$ \\
\hline
\end{tabular}


features are not detectable for diabetic patients. Hence, for detection of glaucoma, the OCT method is chosen. Through the OCT method, in addition to CDR, cup depth and retinal thickness values are calculated for detection of glaucoma. Hence, the features from both fundus image and OCT are considered for glaucoma detection irrespective of the condition of the subject.

\section{SELECTION OF DOMINANT FEATURES}

The computed CDR values from the fundus and OCT images are similar. The mean error of the CDR in fundus image is $1.67 \%$ and for the OCT image, it is $2.75 \%$ respectively. Hence, CDR feature from fundus image is taken as the dominant feature for the classifier. The remaining features such as ISNT ratio for neuro-retinal rim, ISNT ratio for blood vessels along with the two features of OCT method namely cup depth and retinal thickness are applied to the classifier.

\section{DETECTION OF GLAUCOMA BY CLASSIFIER}

In this study one hundred and fifty Fundus and OCT images were used in which 50 were from normal and 100 from glaucoma patients. Out of the 50 normal images, 35 imageswere used for training the network and the remaining 15, were used for testing the network In glaucoma images, 50 images were used for training the network and the remaining 50 images were used for testing the network. The result of the BPN Classifier was $89.33 \%$. The classifier accuracy is inferred to be less, and in order to improve this, the SVM classifier wasused The result of the classifier was $93.66 \%$. Features, such as CDR, ISNT ratio for neuro retinal rim,ISNT ratio for blood vessels, cup depth and average retinal thickness were computed for both sets of samples using the proposed algorithm. The parameters described in section 3.3 were used for the classifier settings for BPN and SVM classifiers. Other parameters such as sensitivity, specificity, positive predictive value and accuracy defining the efficiency of the classifier were also computed here. Table 5 shows the analysis of the classification results.

Table 6 shows the analysis of results of the classifiers with features from fundus images alone, from OCT alone and when the features from both modalities are given collectively to BPN and SVM.

By combining the features, the sensitivity of the SVM classifier is increased to $98 \%$ showing the efficiency of the proposed approach to detect glaucoma. Also, the accuracy of the BPN classifier was $90.76 \%$ and of the SVM classifier, $96.92 \%$. This shows that the combination of features from both fundus and OCT images increases the accuracy of the classifier.

\section{CONCLUSION}

An automated method for the detection of glaucoma in retinal fundus images and OCT images was investigated. Such computerized systems would be very useful for diagnosing glaucoma in the process of mass screening for glaucoma. In this study, new approaches to extracting features from the fundus and OCT images were proposed and used for classification. In fundus image, segmentation of optic disc is done by K-means clustering and the $\mathrm{K}$ value is automatically computed by a hill climbing algorithm. The optic cup is segmented by three methods namely K-means clustering, fuzzy C-means clustering, and spatially weighted fuzzy C-means clustering. In this way, the CDR is calculated from the developed algorithm. From the results, it was inferred that the SWFCM method of segmentation of cup and disc gives less error compared to gold standard values. Blood vessels are segmented by entropy level thresholding. Based on the segmented blood vessels and neuro retinal rim, the corresponding ISNT ratios are calculated. The CDR, ISNT ratio features for diabetic patients are not detected through the fundus image method since cup and disc region merges. Hence, CDR calculation is very difficult using fundus images. To overcome this, OCT images are considered for the detection of glaucoma.

The RV boundary and RC boundary are segmented

Table 5. Analysis of classification results (Combined features).

\begin{tabular}{lcccccccc}
\hline Classifier & TN & TP & FP & FN & Sensitivity & Specificity & Positive Predictive value & Accuracy \\
\hline BPN & 12 & 47 & 3 & 3 & $94 \%$ & $80 \%$ & $94 \%$ & $90.76 \%$ \\
SVM & 14 & 49 & 1 & 1 & $98 \%$ & $93.33 \%$ & $97.91 \%$ & $96.92 \%$ \\
\hline
\end{tabular}

Table 6. Analysis of results.

\begin{tabular}{|c|c|c|c|c|c|}
\hline Classifier & Features from & Sensitivity & Specificity & $\begin{array}{c}\text { Positive Predictive } \\
\text { Value }\end{array}$ & Accuracy \\
\hline \multirow[t]{3}{*}{ BPN } & Fundus image & $92 \%$ & $86.66 \%$ & $95.58 \%$ & $90.7 \%$ \\
\hline & OCT & $92 \%$ & $80 \%$ & $93.87 \%$ & $89.27 \%$ \\
\hline & Combined & $94 \%$ & $80 \%$ & $94 \%$ & $90.76 \%$ \\
\hline \multirow[t]{3}{*}{ SVM } & Fundus image & $96 \%$ & $93.33 \%$ & $97.95 \%$ & $95.38 \%$ \\
\hline & OCT & $94 \%$ & $93.33 \%$ & $97.91 \%$ & $93.83 \%$ \\
\hline & Combined & $98 \%$ & $93.33 \%$ & $97.91 \%$ & $96.92 \%$ \\
\hline
\end{tabular}


in the OCT images by morphological technique. From these boundaries, the CDR value is determined. The success rate of this technique is $78 \%$. In order to increase the accuracy, wavelet transform algorithm is used for the RV boundary and multi-level thresholding algorithm for the RC boundary segmentation. Cup depth and retinal thickness, are also calculated from the OCT image. The SVM classifier applied for these featuresgives maximum accuracy of $93.83 \%$. These CDR, ISNT ratios from fundus image cup depth and retinal thickness features from OCT image are collectively given as input to the classifier. Diagnosic accuracy is improved in SVM to a value of $96.69 \%$ by this form of procedure.

To increase the accuracy further, more work can be done on the following to enhance the accuracy in detection of glaucoma.

1. Higher resolution of fundus image may be used for future work to increase the level of accuracy.

2. RNFL defect and thickness may be used for future work to predict glaucoma.

Acknowledgement: Author contributions: GB: literature search and manuscript writing, data collection, statistical analysis, figures; SS: study design and data interpretation; RV: data analysis; SS: final approval.

Conflict of interest statement: None declared.

\section{REFERENCES}

1. George R, Vijaya L. First World Glaucoma day, March 6, 2008: tack ling glaucoma challenges in India. Indian Journal of Ophthalmology 2008;56(2):97-8

2. Harizman N, Chiang CA, Marmor CM, Jeffrey RR, Liebmann M. The ISNT rule and differentiation of normal from glaucomatous eye. Archophthalmol/American Medical Association 2006;124:1579-83.

3. Herzog A, Boyer KL, Roberts C. Extracting the optical disc endpoints in optical coherence tomography data. In: Proceedings of the 7th IEEE workshops on Application of computer vision, IEEE computer society, Washington, DC, USA, 2005 vol. 1, pp. 263-8.

4. Boyer KL, Herzog A, Roberts C. Automatic Recovery of the Optic Nerve head Geometry in Optical Coherence Tomography. IEEE Transactions on Medical Imaging 2006;25(5):553-70. doi: 10.1109/ TMI.2006.871417

5. Ganeshbabu TR, Satishkumar R, Venkatesh R. A Fast and Reliable Method for early Detection of Glaucoma. Int J Pharm Sci Rev Res 2015;46:258-62.

6. Siddalingaswamy PC, Prabhu KG, Jain V. Automatic Detection and Grading of Severity Level in Exudative Maculopathy. Biomed Eng Appl Basis Commun 2013;23(3):173-9.

7. Liu J, Wong DWK, Lim JH, Jia X, Yin F, Li H, Xiong W, Wong TY. Optic Cup and Disk Extraction from Retinal Fundus Images for Determination of Cup-to-Disc Ratio. In: 3rd IEEE Conference on Industrial Electronics and Applications, ICIEA, 2008 pp. 1828-32.

8. Zhang Z, Liu J, Wong WK, Tan NM, Lim JH, Lu S, Li H. Convex Hull Based Neuro-Retinal Optic Cup Ellipse Optimization in Glaucoma Diagnosis. In: 31st Annual International Conference of the IEEE EMBS Minneapolis, Minnesota, USA, 2009 pp. 1441-4.

9. Wong DW, Liu J, Lim JH, Tan NM, Zhang Z, Lu S, Li H, Teo MH, Chan $\mathrm{KL}$, Wong TY. Intelligent fusion of cup to disc ratio determination methods for glaucoma detection in ARGALI. In: Engineering in Medicine and Biology Society, 2009. EMBC 2009. Annual International Conference of the IEEE 2009:5777-80. doi: 10.1109/ IEMBS.2009.5332534.

10. Nayak J, Rajendra Acharya U, Subbanna Bhat P, Shetty N, Lim TC. Automated Diagnosis of Glaucoma Using Digital Fundus Images. Journal of Medical systems 2009;33(5):337-46.
11. Joshi GP, Sivaswamy J, Krishnadas SR. Optic Disk and Cup Segmentation from Monocular Colour Retinal Images for Glaucoma Assessment. IEEE Transactions on Medical Imaging 2011;30(1):1192205.

12. Mishra M, Nat MK, Dandapat S. Glaucoma Detection from Color Fundus Images. International Journal of Computer \& Communication Technology (IJCCT) 2011;.2(VI):7-10.

13. Ho CY, Pai TW, Chang HT, Chen HY. An automatic fundus image analysis system for clinical diagnosis of glaucoma. In: International Conference on Complex, Intelligent, and Software Intensive Systems, CISIS 2011, pp. 559-64.

14. Kanungo T, Mount DM, Netanyahu NS, Piatko CD, Silverman R, Wu AY. An efficient k-means clustering algorithms: analysis and implementation. IEEE Transactions on Pattern Analysis and Machine Learning 2002;24(7):881 - 92. doi: 10.1109/TPAMI.2002.1017616

15. Walter T, Klein JC. Automatic analysis of colour fundus photography and its application to the diagnosis of diabetic retinopathy. In: Suri JS, Wilson DL, Laxminarayan S, editors. Handbook of Biomedical Image Analysis, Volume II: Segmentation Models, Part B, Chapter 7. New York: Kluwer Academic/ Plenum Publishers, New York, 2005. p. 315-368.

16. Ohashi T, Aghbari Z, Makinouch A. Hill-Climbing algorithm for the efficient colour-based image segmentation. In: IASTED International Conference on signal processing, Pattern recognition and applications (SPPRA 2003), June 2003.

17. Sopharak A, Uyyanonvara B, Barman S. Automatic exudate detection from non-dilated diabetic retinopathy retinal images using fuzzy cmeans clustering. Sensors 2009;9:2148-61. doi:10.3390/s90302148

18. Fitzgibbon A, Pilu M, Fisher RB. Direct least square fitting of ellipses. IEEE T Pattern Anal 1999;21:476-80.

19. Ganeshbabu TR, Shenbagadevi S. Automatic detection of Glaucoma using fundus image. European Journal of Scientific and Research 2011;59(1):22-32.

20. Chuang KS, Tzeng HL, Chen S, Wu J, Chen TJ. Fuzzy c-means clustering with spatial information for image segmentation. Comput Med Imaging Graph 2006;30(1):9-15.

21. Ganeshbabu TR, Satishkumar R, Rengarajvenkatesh. Segmentation of optic nerve head for glaucoma detection using fundus images. Biomedical and Pharmacology Journal 2014;7(2):1-9.

22. Hossam El-Din MA Khalil, Mohamed Yasser Sayed Saif, Mohamed Osman Abd El-Khalek, Arsany Makar. Variations of Cup-to-Disc Ratio in Age Group (18-40) Years Old. Research in Ophthalmology 2013;2(1):4-9. doi: 10.5923/j.ophthal.20130201.02

23. Chanwimaluang T, Fan G. An efficient blood vessel detection algorithm for retinal images using local entropy thresholding. In: Proceedings of the IEEE International Symposium on Circuits and Systems, 2003, May pp. V21-V24.

24. Hecth-Nelsen R. Neurocomputing. Addison-Wesley, Menlo Park, CA; 1990.

25. Zhang GP, Patuwo BE, Hu YM. Forecasting with artificial neural networks: The state of the art. International Journal of Forecasting 1998;14:35-6

26. Smola AJ, Schölkopf B, Müller KR. The connection between regularization operators and support vector kernels. Neural Networks 1998;11(4):637-49. doi: 10.1016/S0893-6080(98)00032-X

27. Ganeshbabu TR, Shenbagadevi S. Glaucoma diagnosis of morphological processing in optical coherence tomography. In: Proceedings of international conference Computer Engineering and Applications. IPCSIT, IACSIT Press, Singapore, 2009; vol. no 2: pp.343-347.

28. Sur A, Patra N, Chakraborty S, Saha, I. A new wavelet based edge detection technique for Iris imagery. In: Proceedings of the IEEE International Advance Computing Conference, Patiala, India, 2009, 6-7 March; IEEE International 2009 pp. 120-4.

29. Papamarkos N, Gatos B. A New Approach for Multilevel Threshold Selection. Graphical Models and Image Processing 1994;56(5):35770.

30. Kuragano T, Yamaguchi A. A Method to generate freeform curves from a hand drawn sketch. JSCI 2007;5(2):5-11.

31. Ganeshbabu TR, Shenbagadevi S, Venkatesh R. Automatic detection of Glaucoma in OCT image. Journal of applied sciences 2012;12(20):2128-38. 